\title{
Visualisierung und Erweiterung astronomischer Daten
}

\author{
Aufwerten, Rekonstruieren und Visualisieren von astronomischen Daten
}

Andrei Linţu $\cdot$ Andreas Steinel

\section{Einleitung}

Die Astronomie ist seit jeher ein interessantes Gebiet. So hat sie auch in der Welt der Informatik viele Begeisterte gefunden - auch unter den Informatikern des Max-Planck-Institutes für Informatik (MPII) in Saarbrücken. Aus der Verquickung von Hobby und Forschungsarbeit - was kann es Naheliegenderes und Schöneres geben? - entstand eine Vielzahl interessanter Forschungsarbeiten. Eine Auswahl werden wir nun an dieser Stelle vorstellen.

Die erste Arbeit beschäftigt sich mit der computergestützten Erweiterung eines Teleskops. Danach stellen wir Verfahren vor, mit deren Hilfe sich $3 \mathrm{D}$ Modelle von Nebeln rekonstruieren lassen und diese auch visualisiert werden können. Abschließend beschreiben wir eine Methode, die dazu dient, astronomische HDR-Aufnahmen zu verbessern und die Zeit des Bildbearbeitenden zu reduzieren.

Als Ansporn und Inspiration über die intellektuelle Arbeit hinaus wurde 2006 ein Observatorium auf dem Dach des Institutsgebäudes errichtet. Dieses Observatorium beherbergt ein $10^{\prime \prime}$ Meade LX200 (2500 m/f10) Schmidt-Cassegrain-Teleskop in einer 2,6-Meter-Kuppel von Baader (vgl. Abb. 1). Das Teleskop wurde für das Augmented-AstronomicalTelescope-Projekt und als Aufnahmeinstrument für die HDR-Bilder benutzt.

\section{Augmented Astronomical Telescope}

Computergestützte GoTo-Teleskope sind heutzutage erschwinglich geworden und weit verbreitet. Fasziniert von den beeindruckenden Aufnahmen, wie sie beispielsweise das Hubble-Weltraumteleskop liefert, erwarten viele unerfahrene Astronomiebegeisterte, auch beim Blick durch ein kleines Teleskop

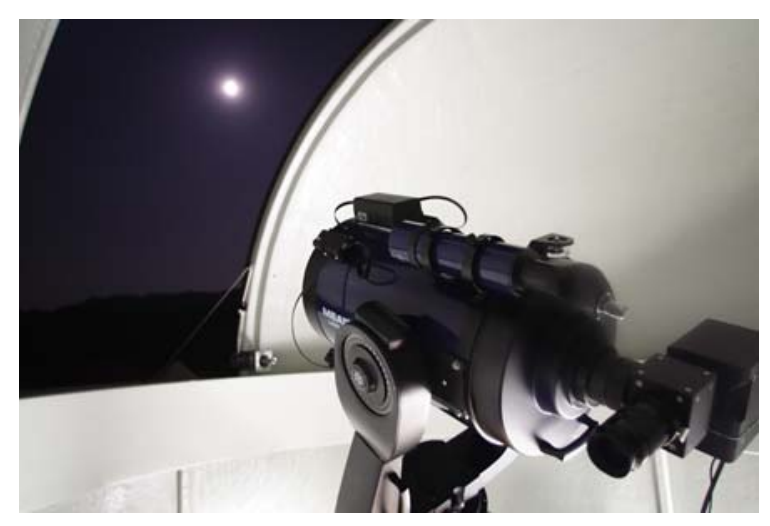

Abb. 1 Die Sternwarte des Max-Planck-Institutes für Informatik mit dem Augmented Astronomical Telescope

solche wunderschönen Bilder zu sehen. Erfolge stellen sich allerdings nur selten sofort ein, denn nur eine kleine Zahl sehr heller Objekte wie beispielsweise der Mond, der Saturn, der Jupiter oder der Orion-Nebel sind auch durch kleine Teleskope gut erkennbar. Objekte von schwächerer Lichtstärke wie Galaxien, planetarische Nebel, Reflexionsnebel oder Sternhaufen, erscheinen zumeist nur als unerkennbare Lichtflecken im Okular des Teleskops.

Als Hilfsmittel für den Hobby-Astronomen kreierten wir ein Erweitertes-Realitäts-System 


\section{Zusammenfassung}

Dieser Artikel beschreibt Techniken, die am Max-Planck-Institut für Informatik in Saarbrücken als Kombination von Informatik und Astronomie entstanden sind. Dabei behandeln wir das computergestützte Augmented Astronomical Telescope, die Rekonstruktion und Visualisierung von planetarischen Nebeln, sowie Reflexionsnebeln wie auch die Anwendung von High-Dynamic-Range-Prinzipien (HDR) der Informatik in der Astronomie und deren Einfluss auf die Bearbeitungsdauer von astronomischen Bildern.

(Augmented Astronomical Telescope) [2], das die Sicht durch das Okular eines Teleskops durch eine Projektion von Information über das aktuell sichtbare Objekt ergänzt. Sowohl lange belichtete Bilder des aktuell beobachteten Objekts, als auch zusätzliche Informationen wie Helligkeit, Entfernung oder Position am Himmel werden projiziert. Für ausgewählte Objekte sind auch Animationen verfügbar.

\section{Aufbau}

Das Projektionsmodul steht jederzeit mit dem Teleskop und einem tragbaren Rechner in Verbindung, sodass die aktuelle Position des Teleskops immer zur Verfügung steht und das projizierte Bild automatisch aktualisiert werden kann. Das System ist nicht als Ersatz der klassischen Deep-Sky-Beobachtung gedacht, sondern vielmehr für Weiterbildungszwecke und als visuelle Hilfe für Einsteiger. Ein Beispiel, wie es benutzt werden kann, ist die Beobachtung eines schwachen Nebels: Das System ermöglicht es, das projizierte Bild mit dem beobachteten Objekt zu vergleichen. Dies verhilft zu einer besseren Wahrnehmung. Da alle Objekte klar gekennzeichnet sind, ist unser System auch sehr hilfreich beim Beobachten von überfüllten Sternfeldern oder Planeten mit mehrere Monden wie z. B. die Monde des Jupiter, die ihre relative Position schnell ändern und dadurch eine genaue Identifizierung des Objektes erschweren.

Unser System (Abb. 2) besteht aus folgenden Bauteilen: einem GoTo-Teleskop, einem speziell entwickelten Projektionsmodul und einem tragbaren Rechner. Da die Planetariums-Software jederzeit über die aktuelle Position des Teleskops

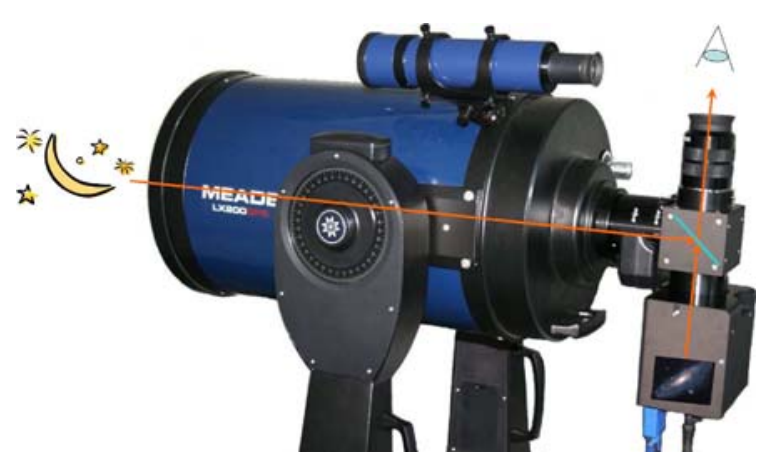

Abb. 2 In der Abbildung sind die Lichtwege des Augmented Astronomical Telescopes dargestellt (orange). Auf das Teleskop ist rechts das Projektionsmodul montiert und kombiniert das Licht von beobachteten astronomischen Objekten mit dem projizierten Bild

verfügt, wird das Projektionsmodul, mit dem jeweils der Sichtrichtung des Teleskops entsprechenden Himmelsteil, aktualisiert.

Die Software basiert auf der Open-SourcePlanetariums-Software Stellarium [1], welche aus einer Datenbank der Planeten unseres Sonnensystems, einer großen Zahl Sterne unserer Galaxie und interessanten Deep-Space-Objekten besteht. Für viele dieser Objekte stehen auch hochauflösende Bilder oder sogar Animationen zur Verfügung, die während des Beobachtens eingeblendet werden können. Der Benutzer kann die gewünschten Informationen und die aktuelle Vergrößerung sowie beispielsweise den Typ des aktuell verwendeten Okulars in einem Menü auswählen.

Ein DLP-basiertes (digital light processing) Projektionsmodul wurde speziell für dieses System entwickelt, um den vorhandenen Kontrast zu maximieren. Herzstück des Moduls ist dabei ein 0.7" Diagonal Digital Micromirror Device (DMD) von Texas Instruments, das in Zusammenarbeit mit Op-Sys Project Consulting entwickelt wurde. Damit wird gewährleistet, dass auch bei schwachen Lichtverhältnissen ein guter Kontrast erzielt werden kann, was für visuelle astronomische Anwendungen sehr wichtig ist und die optische Wahrnehmung vereinfacht.

Um das projizierte Bild mit dem Teleskopbild zu kombinieren, wird ein halbdurchsichtiger Spiegel, ein sogenannter Beamsplitter, benutzt. Ein erweitertes Bild, wie es in Abb. 3 zu sehen ist, entsteht. Dabei werden 90\% des Sternlichts und 10\% des Projektorlichts durch den Spiegel gelassen, um gute Ergebnisse zu erzielen. 


\section{Abstract}

This article describes techniques and methods which were developed at the Max Planck Institute for Informatics, as a combination between astronomy and computer science. We describe the augmented astronomical telescope, the reconstruction and visualisation of reflection and planetary nebulae and a method which applies high dynamic range imaging for astronomical imaging.

\section{D-Nebelvisualisierung und -Rekonstruktion}

$3 \mathrm{D}$-Modelle von astronomischen Objekten gewinnen heutzutage immer größere Bedeutung. Zum einen werden solche Modelle von professionellen Astronomen benutzt, um ihre Theorien unter Beweis zu stellen, und zum anderen gibt es kaum noch eine Planetariumsvorführung ohne einen 3D-Nebel-Rundflug. Allerdings werden die bei den Planetariumsvorführungen verwendeten $3 \mathrm{D}$ Modelle meist von Künstlern entwickelt und haben mit der physikalischen Realität wenig zu tun.

Reflexionsnebel sind von einem oder mehreren Sternen beleuchtete Staubwolken im Weltraum. Das abgestrahlte Sternlicht wird von diesen Staubwolken gestreut und absorbiert, wodurch die Erscheinung dieser Nebel, abhängig vom Blickwinkel, sehr unterschiedlich ist. Mit unserem physikalisch korrekten Visualisierungssystem [6], das den Lichttransport in
Reflexionsnebeln simuliert, kann nun eine EchtzeitVisualisierung des 3D-Nebelmodells aus beliebigen Blickwinkeln erzeugt werden.

Das System zieht Streuung und Absorption in Betracht, um ein physikalisch korrektes Rendering zu erreichen. Dabei besteht jedes Nebelvolumen hauptsächlich aus mehreren Volumenelementen (Voxeln), die jeweils eine bestimmte Staubdichte aufweisen. Auf der Voxelebene wird sogar Mehrfachstreuung berücksichtigt. Für das Rendering der Datensätze wird ein Graphics Processing Unit (GPU)-basiertes Ray-casting-Verfahren verwendet.

Basierend auf der astrophysikalischen Literatur über die Staubverteilung in Reflexionsnebeln benutzen wir ein beschränktes $3 \mathrm{D}$-Perlin-RauschModell um unsere synthetischen Nebelvolumen zu generieren. Das 3D-Rausch-Modell entsteht aus der Kombination mehrerer Rauschfunktionen mit unterschiedlichen Amplituden und Frequenzen. Durch die Variation verschiedener Parameter können Nebel mit unterschiedlichen Morphologien generiert werden, wie in Abb. 4 zu sehen ist.

Ein Hauptziel unserer Arbeit war es, glaubhafte $3 \mathrm{D}$-Staubverteilungen für gegebene Nebel basierend auf Nebelbildern zu erzeugen. Dabei gilt zu beachten, dass für die Rekonstruktion von astronomischen Objekten im Normalfall nur unzureichende Informationen vorhanden sind: Wie bei unterbestimmten Gleichungssystemen sind mehrere Lösungen möglich und prinzipiell auch plausibel. Ausschließen könnte man diese nur mit Bildern aus einem anderen Blickwinkel, die jedoch naturgemäß nicht
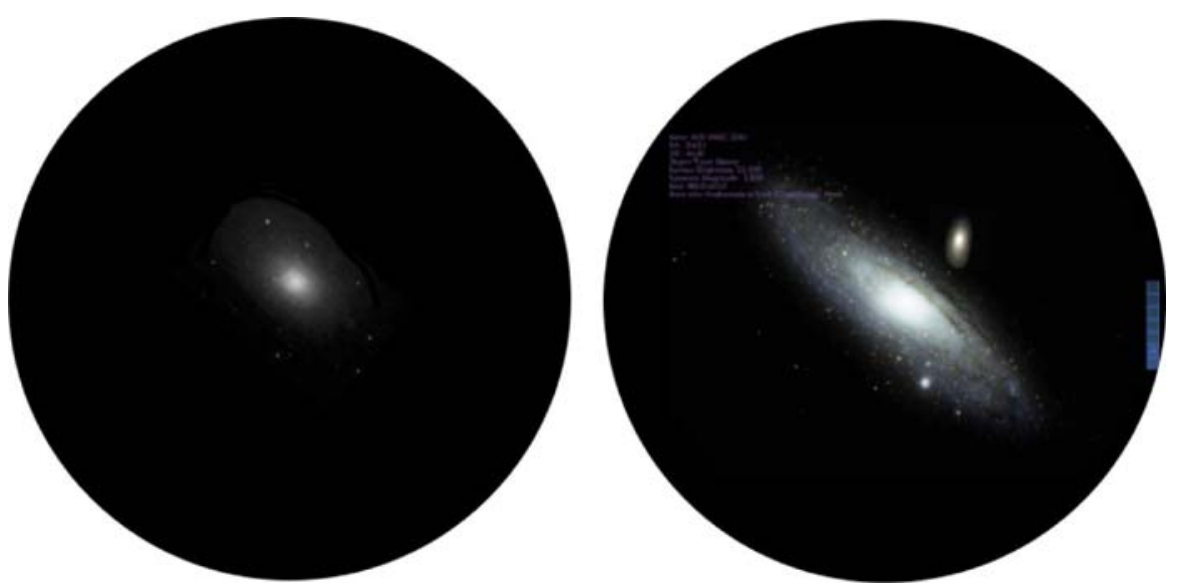

Abb. 3 Links ist die „„normale" Sicht durch das Okular eines gewöhnlichen Teleskops beim Beobachten der Andromeda-Galaxie (M31) zu sehen. Auf der rechten Seite befindet sich das erweiterte Bild. Der Beobachter kann sich durch das erweiterte Bild eine viel bessere Vorstellung vom Aussehen des Objektes machen und erhält gleichzeitig zusätzliche Informationen 

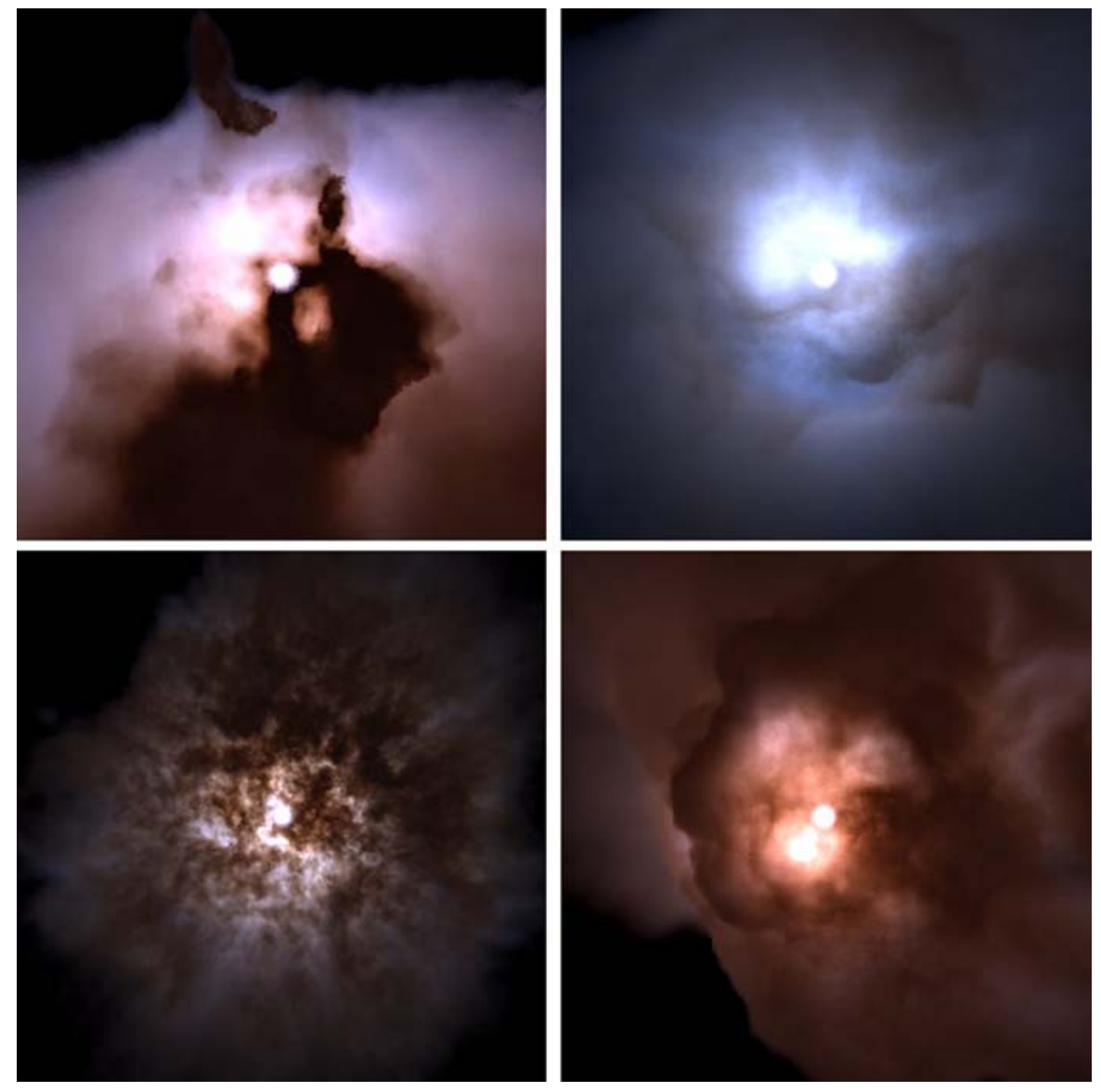

Abb. 4 Rendering
verschiedener
synthetischer $3 D$ -
Reflexionsnebelvolumen
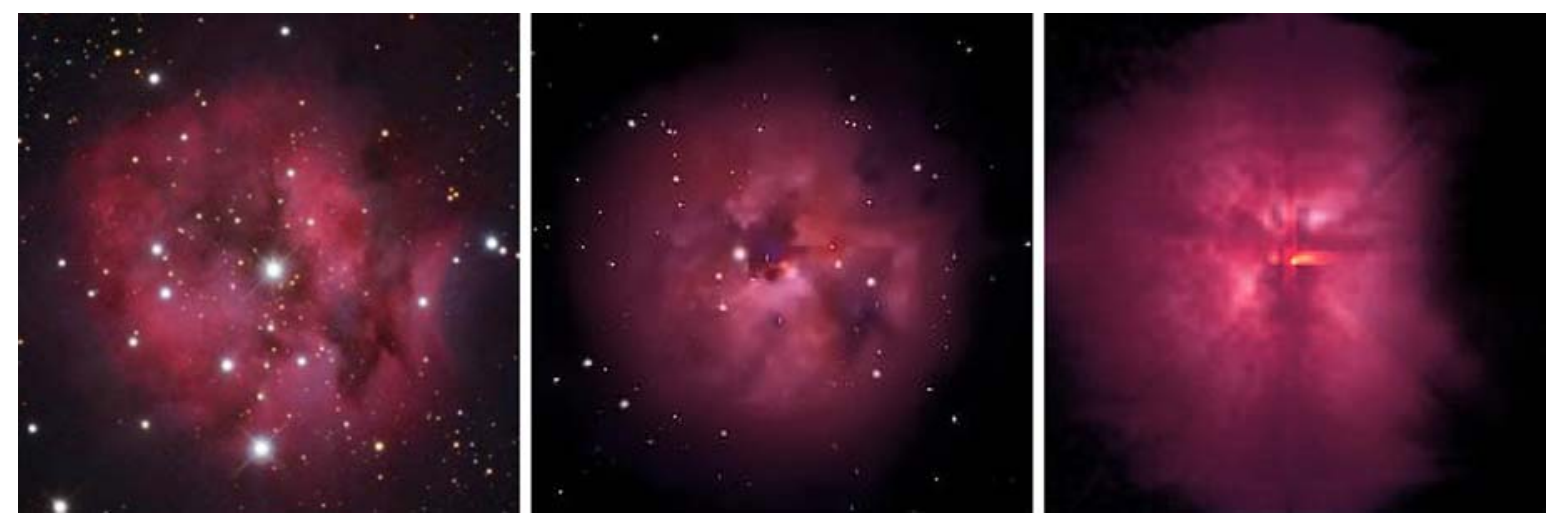

Abb. 5 Links: Bild des Kokon-Nebels. Mitte: Frontansicht des rekonstruierten Nebels. Rechts: Seitenansicht des rekonstruierten Nebels

zur Verfügung stehen. Allerdings ist es möglich, durch sorgfältige Führung des Rekonstruktionsprozesses $3 \mathrm{D}$-Staubverteilungen für gegebene Nebel zu rekonstruieren [4].

Durch ein nichtlineares Rekonstruktionsverfahren, kombiniert mit einer optimierten Traversierung des Volumens, erhalten wir 3D-Nebelmodelle, die, obschon nicht vollkommen exakt, zumindest sehr glaubwürdig sind. Die Optimierung erfolgt durch ständiges Vergleichen des Eingangsbildes mit dem gerenderten Modell und basiert auf dem Minimieren einer Fehlerfunktion. Zur Verbesserung der Rekonstruktionsergebnisse wird das Volumen vom Zentrum nach außen sukzessive in konzentrischen Schalen durchlaufen. Ein Beispiel ist in Abb. 5 für den Kokon-Nebel zu sehen. 
Eine andere Klasse interessanter astronomischer Objekte sind planetarische Nebel. Diese Sorte von Nebeln entstehen, wenn Sterne - wie unsere Sonne - ihren Lebenszyklus beenden. Diese Sterne sind nicht groß genug, um als Supernova zu explodieren, sondern gehen durch einen sogenannten Roter-Riese-Abschnitt, in dem sie stark expandieren. Diese Phase ist vergleichbar kurz und instabil und der Stern kollabiert zu einem weißen Zwerg. Die Gase, die durch die Roter-Riese-Phase ausgestoßen wurden, werden nun von dem weißen Zwerg ionisiert und der Nebel beginnt zu leuchten: die Geburt eines planetarischen Nebels. Aufgrund ihrer spezifischen Entstehung haben viele planetarische Nebel einen achsensymmetrischen Aufbau.

Im Max-Planck-Institut für Informatik ging es in mehreren Forschungsprojekten darum, Wege für die 3D-Rekonstruktion planetarischer Nebel zu finden. Von Magnor et al. [5] wurde ein Verfahren entwickelt, mit dem die Komplexität des Rekonstruktionsprozesses für die Gasverteilung in planetarischen Nebeln durch Ausnutzung der inhärenten Achsensymmetrie minimiert wird. Dieses Verfahren wurde von uns erweitert [3], um nicht nur die Gasverteilung, sondern gleichzeitig auch die Staubverteilung zu rekonstruieren. Andere Gruppen, beispielsweise Wenger et al. [12] verwenden schnellere, linear-algebraische Rekonstruktionsansätze, die auch Asymmetrien in planetarischen Nebelmodellen in Betracht ziehen.

\section{Tonemapping für astronomische Bilder}

Seit mehr als einer Dekade gibt es in der Bildverarbeitung die Begriffe high dynamic range (HDR) und Tonemapping [8]. Hinter diesen Begriffen verbergen sich Verfahren, die sich mit der Aufnahme und der Darstellung von Szenen mit extremen Lichtverhältnissen befassen. Die meisten heutzutage verwendeten Digitalkameras sind in ihrem Dynamikumfang so stark eingeschränkt, dass es nicht möglich ist, einige Szenen komplett einzufangen und später am Rechner naturgetreu darzustellen. Viele Bilder, mit denen wir täglich arbeiten, sind auf den Computer abgestimmt und weisen einen Informationsgehalt von 8 Bit pro Farbkanal auf, welcher auch dem Standarddarstellungsbereich eines Computermonitors entspricht. Dadurch ist es nicht möglich, Szenen mit hohem Dynamikumfang darzustellen.

Das beste Beispiel aus dem Alltag ist das Fotografieren in einem Raum, in dem sich auch ein
Fenster im Bildbereich befindet: Entweder ist der Raum zu dunkel und die Außenwelt sichtbar, oder der Raum ist hell erleuchtet und es sind keine Details im überbelichteten Fenster zu erkennen. Beides zu sehen ist bisher nur dem (menschlichen) Auge vorbehalten, welches einen logarithmischen und keinen linearen Dynamikbereich besitzt, wie er bei Kameras üblich ist.

Genau an diesem Punkt setzten nun HDR-Verfahren an, die mehrere Bilder mit unterschiedlicher Belichtung (meistens Verdopplung der Belichtungszeit von Bild zu Bild) zu einem Bild mit hohem Dynamikumfang umrechnen. Damit wird versucht, die Realität genauer nachzubilden. Neu ist diese Technik nicht, wurde sie doch schon vor mehr als einem halben Jahrhundert verwendet - damals allerdings noch auf Analogfilm und durch geschicktes Überblenden von verschieden belichteten Bildern. Das Verfahren ist als Abwedeln (engl. dodge and burn) bekannt.

Durch Techniken wie das gängige RobertsonVerfahren [9] kann die Belichtungsserie automatisch zu einem HDR-Bild mit oft extrem gesteigertem Dynamikumfang rekonstruiert werden. Allerdings ist dieses dann nicht mehr komplett auf einem normalen Computermonitor darstellbar. An diesem Punkt kommt das Tonemapping ins Spiel: Aus dem HDR-Bild soll wieder ein Bild erzeugt werden, das dem Ergebnis des HDR-Verfahrens möglichst nahe kommt, aber auf einem Bildschirm darstellbar ist. Die Anzahl der verschiedenen Verfahren ist groß, jedoch liefert keines von ihnen für jedes mögliche Bild ein perfektes Ergebnis. Oft wird daher das Tonemapping als künstlerische Anwendung angesehen, die mittlerweile auf Fotoplattformen wie z. B. Flickr stark vertreten ist.

$\mathrm{Zu}$ den einfachsten Tonemappern gehört der logarithmische Tonemapper, der den natürlichen Logarithmus jedes Pixels berechnet und somit den Dynamikumfang analog zum menschlichen Auge wieder reduziert. Allerdings ist der Logarithmus ein globaler Operator, der jeden Pixel gleich behandelt, was oft nicht die gewünschten Ergebnisse liefert. Im Allgemeinen versucht man durch Tonemapping den Kontrast in einem Bild so zu verändern, dass der minimale und der maximale Farbwert noch auf den Farbraum abgebildet werden können (globaler Kontrast) und dabei der lokale Kontrast erhalten bleibt. 
Die Schritte des Akquirierens, Rekonstruierens und Tonemappens bilden zusammen die HDR-Pipeline.

\section{Bezug zur Astronomie}

In klaren Nächten erkennen wir - gute Lichtverhältnisse vorausgesetzt - neben dem Mond und unseren sieben Nachbarplaneten noch eine nicht unerhebliche Anzahl an Sternen am Himmel. Bei den Sternen, die mit freiem Auge erkennbar sind, handelt es sich allerdings nur um die hellsten. Erst bei Aufnahmen von mehreren Minuten, Stunden oder manchmal sogar Tagen kommen unscheinbarere und schwächere Objekte zum Vorschein. Ein Nachteil der langen Belichtungszeit ist allerdings, dass sich helle Sterne in der Nähe der schwachen Objekte zu riesigen hellen Scheibchen aufblähen, bedingt durch den enormen Helligkeitsunterschied.

Die Bilder des Hubble-Weltraumteleskops werden oft nur mit fester Belichtungszeit aufgenommen. Allerdings kann man sie dennoch eingeschränkt als HDR-Bilder betrachten, da die Bilder meist 16 oder 32 Bit an Farbinformationen pro Kanal aufweisen. Diese höhere Farbtiefe wirkt dem oben genannten Effekt entgegen. Dennoch kann die auftretende Dynamik oft nicht komplett aufgenommen werden.

Amateurastronomen müssen jedoch zumeist auf Belichtungsserien zurückgreifen. Einzelne Aufnahmen mit extrem langer Belichtungszeit, womöglich noch mit einer HDR-Kamera, sind oftmals nicht möglich, da HDR-Kameras auf der einen Seite noch recht teuer sind, und auf der anderen Seite die atmosphärischen Bedingungen diese Aufnahmen oft gar nicht erlauben.

Bei fast allen im Internet oder bei Zeitschriften erscheinenden astronomischen Bildern ist daher sehr viel Nachbearbeitung in Form von Handarbeit notwendig. Die Befragung von einigen bekannten Amateur- und Berufsastronomen ergab, dass die verschieden belichteten Bilder meist als einzelne Ebenen in Photoshop bearbeitet werden. Der Prozess kann einen Zeitraum von mehreren Stunden bis zu einigen Tagen in Anspruch nehmen.

Unsere Idee war nun [11], die bekannten Verfahren aus der Informatik auf astronomische Bilder anzuwenden und zu sehen, ob sie damit auch funktionieren und ggf. die Bearbeitungszeit in Photoshop durch die Zuhilfenahme von bereits existierenden Techniken enorm reduzieren. Mangels passenden Ausgangsmaterials haben wir eigens für diesen
Zweck im Observatorium des MPII Belichtungsreihen aufgenommen. Dabei fanden eine SBIG ST-10 Kamera mit einem 16-bit monochrom-CCD Chip, eine Adaptive Optik sowie das schon beschriebene Meade LX20o Anwendung.

\section{Anwendung der HDR-Prinzipien}

Messier 42, besser bekannt als Orion-Nebel, wurde als Zielobjekt ausgewählt, da der Nebel mit bloßem Auge zu erkennen ist und damit sehr kurze Belichtungszeiten zulässt. Die Belichtungsserie enthält Bilder mit 5, 10, 25, 40, 60 und 120 Sekunden Belichtungszeit. Die Bilder sollten astronomisch kalibriert sein, d. h. Dunkel- und Ausleuchtbild müssen erstellt, sowie mehrere Aufnahmen kombiniert werden, um somit die Artefakte von kosmischen Strahlen und das Rauschen zu vermindern. Erst danach kann aus den Einzelbildern ein HDR-Bild rekonstruiert werden.

Die Untersuchung verschiedener TonemappingVerfahren $[7,8]$ ergab, dass die Bilder grundsätzlich besser aussehen, jedoch - abhängig vom Tonemapper - verschiedene Artefakte um die Sterne herum entstehen (vgl. Abb. 7 links). Die Sterne erschienen nach der Anwendung der Verfahren zumeist breiter und größer. Die Erklärung ist einfach: Jeder Stern wird beugungsbedingt bei der Aufnahme in einer gaussglockenähnlichen Form abgebildet, welche einen steilen Gradienten aufweist. Durch das Tonemapping wird dieser Gradient abgeflacht und der Stern somit visuell breiter.

\section{Lösung des Tonemapping-Problems}

Eine Lösung für dieses Problem ist es, die Sterne getrennt vom (meistens wichtigeren und schöneren) Hintergrund zu betrachten. Hierfür wurde ein bereits bestehendes Verfahren [10] dahingegen erweitert, dass auch Sterne gefunden werden, die in der Helligkeit um viele Magnituden voneinander abweichen. Dabei wird das Bild im Vorfeld mehrfach mittels des Logarithmus-Operators bearbeitet und ermöglicht somit die Sternendetektion mittels Lucy-Richardson-Deconvolution für alle Sterne im Bild. Danach werden die Sterne ausgeschnitten und die entstehenden Hohlräume mittels Inpainting, einer Technik zum intelligenten Füllen von Informationslücken, befüllt. Somit erhalten wir die Hintergrundebene.

Falls die Ausgangsqualität des Bildmaterials zu schlecht ist, kann man die Sternenebene verbessern, 


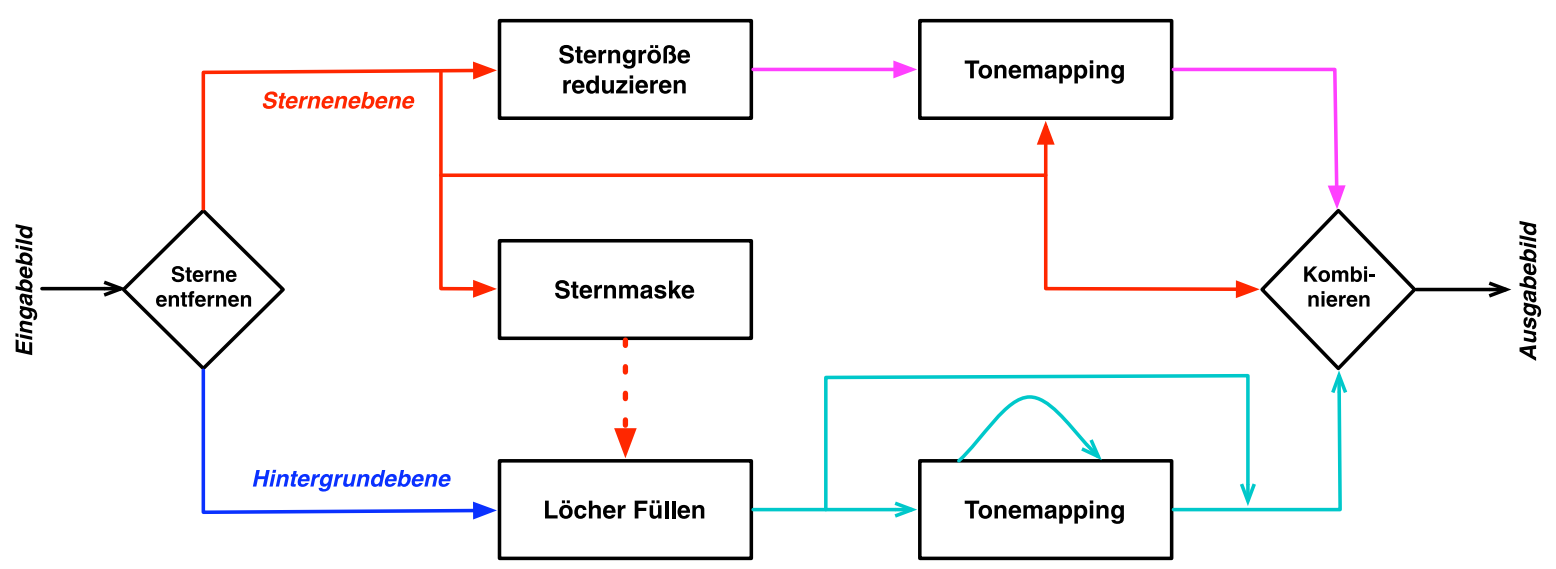

Abb. 6 Übersicht des beschriebenen Systems zum Tonemapping von astronomischen Bildern
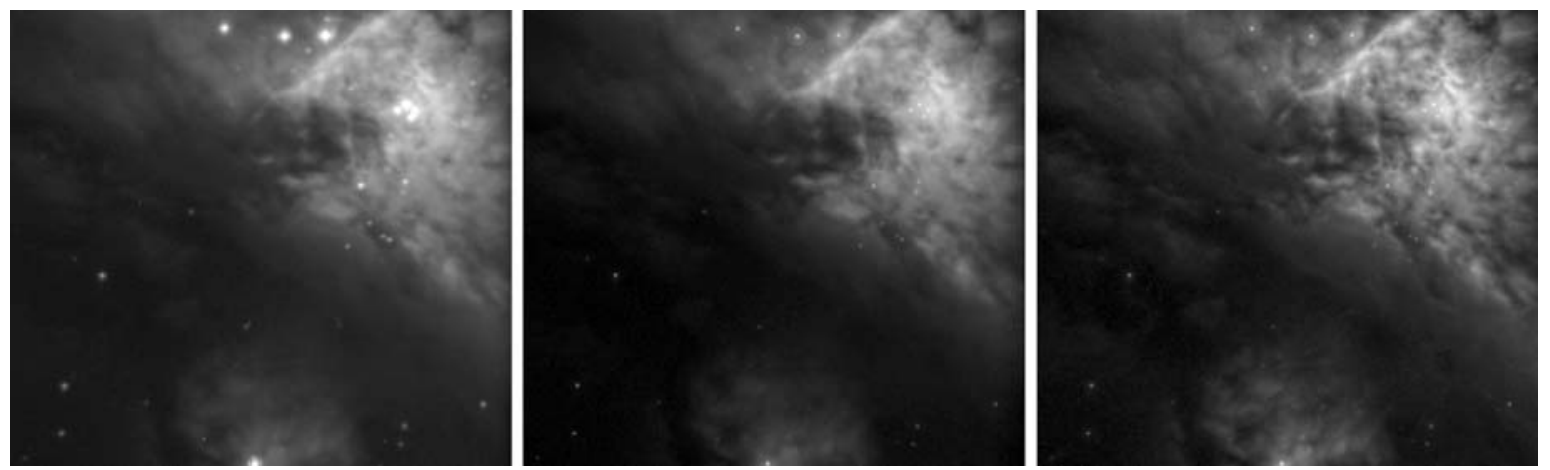

Abb. 7 Bild nach dem Tonemapping mittels Mantiuk Tonemapper (Standardeinstellungen) [7]. Links: Tonemapping ohne unsere Technik. Mitte: einmaliges Tonemapping und Wiederherstellung der Sternenebene. Rechts: gleich wie Mitte, jedoch zweimaliges Tonemapping

indem man die Sterne durch Deconvolution zusammenschrumpfen lässt oder versucht, die Sterne mit exakten Helligkeiten zu rekonstruieren und danach ein neues Sternenfeld mit reduzierter Dynamik zu erzeugen. Die Sternenebene sollte mit einem globalen Tonemapping-Verfahren bearbeitet werden, sodass nur die Helligkeitslevel global angeglichen werden.

Diese zwei separat voneinander bearbeitbaren Ebenen kann man nun wieder vereinen. Zur Verbesserung des Resultates können auch mehrere verschiedene Tonemapping-Verfahren auf die einzelnen Ebenen angewendet werden. Eine Übersicht des gesamten Systems ist in Abb. 6 dargestellt. Abbildung 7 zeigt die Anwendung der beschriebenen Technik, um eine Abbildung des Orion-Nebels optisch aufzuwerten. Das Umwandeln der Eingangsbilder bis hin zum Tonemapping dauert mithilfe von CUDA (Rechnen auf der Grafikkarte) nur wenige Minuten.

\section{Schluss}

In diesem Artikel haben wir einen Einblick in drei unterschiedliche Forschungsbereiche gewährt. Ein Augmented Astronomical Telescope wurde beschrieben, das imstande ist, das durch das Okular eines astronomischen Teleskops sichtbare Bild zu ergänzen. Der Beobachter kann das beobachtete Objekt mit einem hochauflösenden Bild desselben Objekts vergleichen. Die Identifizierung kleiner astronomischer Objekte, beispielsweise von Satelliten von Planeten oder schwachen Sternen, ist deutlich erleichtert. Animationen wie die 3D-Verteilung oder die zukünftige Bewegung des Objekts können direkt im Okular angezeigt werden. Die Grundideen des von uns entwickelten Systems können auch in anderen Gebieten verwendet werden, bei dem die Erweiterung eines optisch vergrößerten Bildes erwünscht ist, wie z. B. in der Mikroskopie.

Ferner haben wir ein Verfahren für die Visualisierung und Rekonstruktion von Reflexionsnebeln 
beschrieben. Die vorgeschlagenen Methoden können um physikalisch korrekte Animationen für astrophysikalische Forschungen oder als Einstieg für $3 \mathrm{D}$-Modelle in Planetariumsvorführungen verwendet werden. Darüber hinaus haben wir kurz über Verfahren für die Rekonstruktion von 3D-Modellen von planetarischen Nebeln berichtet.

Abschließend wurde eine Technik vorgestellt, die das Tonemapping von astronomischen Bildern verbessert, indem die Sterne des Bildes vor dem Tonemapping des Hintergrundes intelligent entfernt werden. Die Sternenebene wird getrennt vom Hintergrund verarbeitet und am Schluss wieder zu einem Bild zusammengefasst. Diese Technik erlaubt es, die Zeit der Bearbeitung in Photoshop zu verkürzen und die Qualität der Bilder zu verbessern.

\section{Literatur}

1. Chéreau F (2006) Stellarium - erhältlich von http://www.stellarium.org/
2. Linț A, Magnor M (2006) An augmented reality system for astronomical observations. In: Proc IEEE Virtual Real Conf 2006, pp 119-126

3. Linţu A, Lensch HPA, Magnor M, El-Abed S, Seidel HP (2007) 3D reconstruction of emission and absorption in planetary nebulae. In: Proc IEEE/EG Int Symp Vol Graph 2007, pp 9-16

4. Linţu A, Hoffmann L, Magnor M, Lensch HPA, Seidel HP (2007) 3D reconstruction of reflection nebulae from a single image. In: Proc Vis Mod Vis 2007, pp 109-116

5. Magnor M, Kindlmann G, Hansen C, Duric N (2004) Constrained inverse volume rendering for planetary nebulae. In: Proc IEEE Conf Vis 2004, pp 83-90

6. Magnor M, Hildebrand K, Linţu A, Hanson A-J (2005) Reflection nebula visualisation. In: Proc IEEE Conf Vis 2005, pp 255-262

7. Mantiuk R, Krawczyk G, Myszkowski K, Seidel HP (2007) High dynamic range image and video compression - fidelity matching human visual performance. In: Proc IEEE Int Conf Image Process 2007, pp 9-12

8. Reinhard E, Ward G, Pattanaik S, Debevec P (2005) High dynamic range imaging: Acquisition, display and image-based lighting. Morgan Kaufmann Publishers, San Francisco, CA, ISBN 978-0125852630

9. Robertson MA, Borman S, Stevenson RL (2003) Estimation-theoretic approach to dynamic range improvement using multiple exposures. J Electron Imaging 12(2): 219-228

10. Snel R (1996) Detecting stars in crowded stellar fields. In: SSAB Symp Image Anal, Proc 1996, pp 106-110

11. Steinel $A$ (2008) HDR acquisition, processing and tone-mapping of astronomical images. Master Thesis, Universität der Saarlandes/Max-Planck-Institut für Informatik (Supervisor: HPA Lensch)

12. Wenger S, Aja Fernández J, Morisset C, Magnor M (2009) Algebraic 3D reconstruction of planetary nebulae. J WSCG 17:1-3 